\title{
Real-time vehicle-to-grid control for frequency regulation with high frequency regulating signal
}

\author{
H. Liu ${ }^{1 *}$, K. Huang ${ }^{1}\left(\mathbb{D}\right.$, Y. Yang ${ }^{1}$, H. Wei ${ }^{1}$ and S. Ma ${ }^{2}$
}

\begin{abstract}
The large-scale popularization of electric vehicles (EVs) brings the potential for grid frequency regulation. Considering the characteristics of fast response and adjustment of EVs, two control strategies of automatic generation control (AGC) with EVs are proposed responding to two high frequency regulating signals extracted from area control error (ACE) and area regulation requirement (ARR) by a digital filter, respectively. In order to dispatch regulation task to EVs, the capacity of regulation is calculated based on maximum V2G power and the present V2G power of EVs. Finally, simulations based on a two-area interconnected power system show that the proposed approaches can significantly suppress frequency deviation and reduce the active power output of traditional generation units.
\end{abstract}

Keywords: Area control error (ACE), Automatic generation control (AGC), Electric vehicle, Frequency regulation, High frequency regulating signal

\section{Introduction}

Electric vehicles (EVs) play a vital role in dealing with fossil energy crises and reducing carbon emissions, because they benefit local and global climates [1]. The large-scale integration of EVs into power grids poses a great challenge to power system operation and control due to the randomness and uncertainty of plug-in time and charging demands of EVs [2]. For instance, large-scale disordered charging can aggravate the peak load level, increase the burden on power lines, and damage the safety of grid operation.

However, the EV batteries, as mobile energy storage devices, can be considered for providing support for the power grid. In 1997, Kempton and Letendre firstly proposed the concept of vehicle to grid (V2G) [3]. Since then, V2G has shown great applications such as voltage regulation $[4,5]$, spinning reserve [6, 7], load peak shifting [8], and frequency regulation [9]. In particular, grid frequency regulation has been paid significant attentions.

Frequency, as one of the most important indexes of power system operation must be controlled within the allowable limits by regulating power sources such as

\footnotetext{
* Correspondence: hughlh@126.com

'College of Electrical Engineering, Guangxi University, Nanning 530004, China Full list of author information is available at the end of the article
}

generators. Because of the fast response characteristics, EV fleet participating in frequency regulation has a natural advantage over other ancillary services. In [10], an energy management algorithm was proposed for EVs to reduce the peak load and simultaneously perform frequency regulation. A primary frequency regulation using EVs was addressed by adaptive droop control in [11]. EVs can also be considered as a kind of regulating resource to take part in supplementary frequency regulation [12]. In [13], an aggregator of EVs was considered to make efficient use of EVs' distributed power to perform optimal dispatch. In [14], the economics of V2G providing regulation services, spinning services, and peak power were analyzed. In [15], high frequency signal was designed for the control center to dispatch the regulation to EVs, but the maximum V2G power was considered as the capacity for regulation. However, in practice, the maximum V2G power cannot represent the real capacity of an EV for regulation, because the capacity of an EV for regulation will vary in real time along with the variation of the charging/discharging power [12]. Almost all existing researches have focused on area control error (ACE), while in real power systems, area regulation requirement (ARR) is considered in the automatic generation control (AGC) 
system. Therefore, it is necessary to discuss and compare ACE and ARR for EVs' participation in regulation.

To ensure the success of frequency regulation, the capacity of EVs should be accurately evaluated. However, the capacity of an EV fleet varies, which increases the difficulty of evaluation. In [16], an approach for estimating achievable power capacity was proposed in a probabilistic manner, where capacity probability distribution was obtained by classifying EV groups and analyzing historical data. In [17], a queueing network of EVs was modeled to estimate the capacity for regulation up during under-frequency and regulation down during over-frequency. In [18], achievable V2G capacity was evaluated by calculating energy and power boundaries.

In this paper, the focus is placed on V2G control for EVs to participate in AGC of power systems. The main contribution of the paper is the proposal of two kinds of high frequency regulating signals generated by $A C E$ and $A R R$ respectively, to provide real-time closed loop V2G control, where the capacity for regulation is calculated based on the maximum V2G power and the present V2G power of EVs. According to the proposed two V2G strategies, the regulation from the control center is dispatched to EVs as much as possible. Simulations on a two-area interconnected power system are used to show the effectiveness of the proposed strategies in improving the quality of system frequency and reducing the output of traditional generating units.

The remainder of this paper is organized as follows. In Section 2, the V2G framework for AGC with EVs is introduced and in Section 3, real-time V2G control strategies are proposed for EVs to take part in AGC. The simulation system is described in Section 4. Simulations are presented and discussed in Section 5. Finally, Section 6 draws the conclusions.

\section{V2G framework for AGC with EVS}

\subsection{Hierarchical control for EVs}

Since the V2G power of an individual EV is not big enough, large numbers of EVs have to be aggregated to take part in the AGC of the power grid. With the aggregation of EVs, a hierarchical control framework, consisting of a dispatching layer, an intermediate layer, and an executive layer, is illustrated in Fig. 1.

The dispatching layer is performed in the control center to dispatch regulation task to EV aggregators of the intermediate layer. In the intermediate layer, the regulation task is sent to charging stations and the EVs' regulation capacity is uploaded to the dispatching layer. Therefore, the intermediate layer is responsible for communication between the dispatching layer and the executive layer. The executive layer manages charging stations to implement the V2G control according to the regulation task.

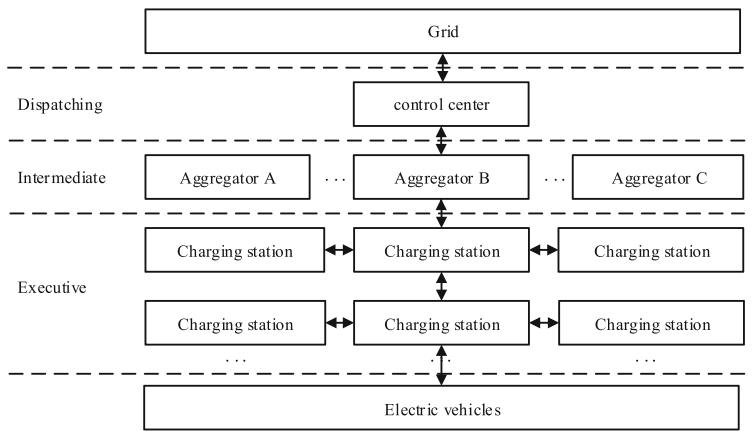

Fig. 1 Hierarchical control of EVs

\subsection{V2G framework for AGC with EVs}

AGC system plays an important role in keeping system frequency within the limits. When EVs participate in AGC system, the framework of AGC with the V2G control of EVs can be illustrated in Fig. 2. As shown in Fig. 2, both the capacity and the regulation signal are the keys to be considered. The capacity related to the charging/discharging of an EV determines how much regulation task should be dispatched to the EV. The regulation signal is based on the capacity and the power-load mismatch and is sent from the control center to the EV. Therefore, the regulation task has to be dispatched within the capacity of the EV.

Because EVs and the traditional generation units undertake the regulation together, the regulation task stemming from the power-load mismatch will be divided into two parts. While EVs undertake this task, the traditional generation units will undertake the remainder of the regulation task.

\section{Methods}

\subsection{Real-time V2G control \\ 3.1.1 Dispatching approach}

\section{(1) ACE and ARR signal}

In the AGC system with EVs, ACE and ARR are two kinds of signals available for performing V2G control. As shown in Fig. 3, ACE is calculated based on the frequency and the line power deviation if tie-line frequency

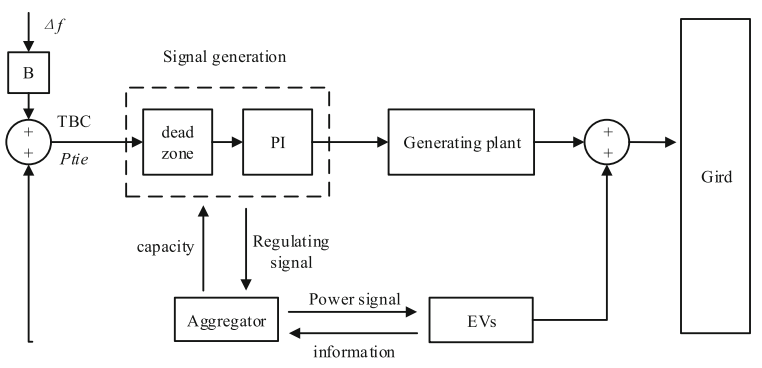

Fig. 2 V2G framework for AGC with EVs 


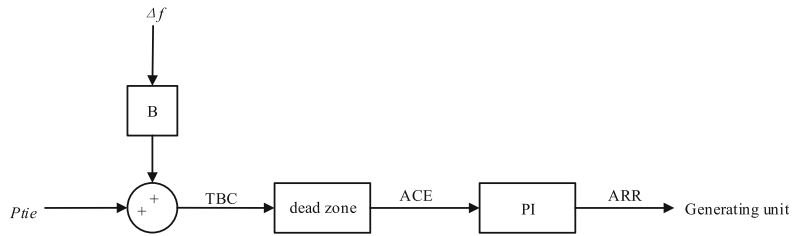

Fig. 3 ACE and ARR signals for regulation

bias control (TBC) is used, while ARR is obtained after ACE through PI controller.

Although both ACE and ARR may be considered as signals for regulation, there are some differences between them. As ACE largely belongs to Gaussian distribution with zero mean, the accumulation of ACE is approximately zero. This means that the increment of EVs' battery energies approach to zero. Therefore, the regulation from ACE will not have negative impact on EVs' battery energy levels. On the contrary, if EVs respond to ARR, their battery energies will deviate from the initial values. This is because the accumulation of ARR is not zero when PI controller is used. However, using ARR has the effect of stabilizing frequency.

Therefore, using ACE and ARR for regulation have their respective advantages and disadvantages.

(2) The control with high-frequency component

The response time of traditional generation units is about $10 \mathrm{~s}$, while EVs can respond to regulation in milliseconds as they are connected to the power grid through power electronics equipment. Therefore, EVs are more suitable for responding to fast regulation signals (i.e., high frequency components of ACE or ARR) compared to traditional generation units. High frequency components account for about $30 \%-40 \%$ of the total components and can be covered by EVs [19].

Through a digital high-pass filter, ACE and ARR can be divided into high frequency components and low frequency components. Therefore, the control loops with high frequency components of ACE and ARR are proposed and shown in Figs. 4 and 5, respectively.

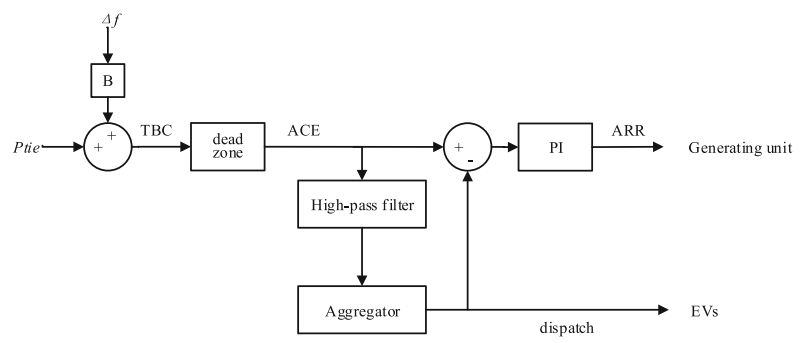

Fig. 4 The control loop with high-frequency components of ACE

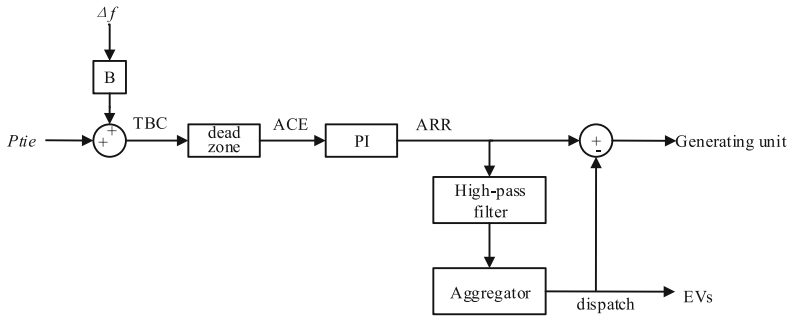

Fig. 5 The control loop with high-frequency components of ARR

\subsubsection{Condition constraint}

EVs participating in regulation need have sufficient time such that the battery energy can be charged to the expected level. Besides, for the safety of EVs' batteries, their state of charge (SOC) levels must be kept within the limits. Therefore, for an EV taking part in AGC, various constraints have to be considered as follows.

\section{(1) Time constraint}

The plug-in time must be sufficient to charge the EV battery level to satisfy the charging demand of the EV owner. The least plug-in time that can be used to achieve the expected battery level of an EV is calculated as:

$$
t_{i}^{\min }=\frac{E_{i}^{\mathrm{r}}\left(\mathrm{SOC}_{i}^{\mathrm{e}}-\mathrm{SOC}_{i}^{\mathrm{in}}\right)}{P_{\max }}
$$

where $E_{i}^{\mathrm{r}}$ is the rated battery capacity of the $i$ th $\mathrm{EV}$, $\mathrm{SOC}_{i}^{\mathrm{e}}$ and $\mathrm{SOC}_{i}^{\mathrm{in}}$ are the expected battery energy level and the battery energy level at the plug-in time, respectively. $P_{\max }$ is the maximum charging rate in charging station.

Considering (1), the time available for frequency regulation has to satisfy the following constraint:

$$
t_{i}^{\mathrm{in}}<t<t_{i}^{\mathrm{d}}-t_{i}^{\min }
$$

where $t_{i}^{\text {in }}$ and $t_{i}^{\mathrm{d}}$ are the plug-in time and expected plugout time of the $i$ th EV, respectively.

(2) Battery energy constraint

To keep battery safety, the limits of EV battery SOC are considered as:

$$
\mathrm{SOC}_{\min }<\mathrm{SOC}_{i, t}<\mathrm{SOC}_{\max }
$$

where $\mathrm{SOC}_{\min }$ and $\mathrm{SOC}_{\max }$ are the minimum and maximum battery SOCs, respectively, and $\mathrm{SOC}_{i, t}$ is the realtime battery SOC.

\subsubsection{Capacity calculation for regulation}

The capacity for regulation of an EV can be calculated according to the maximum V2G power and the 


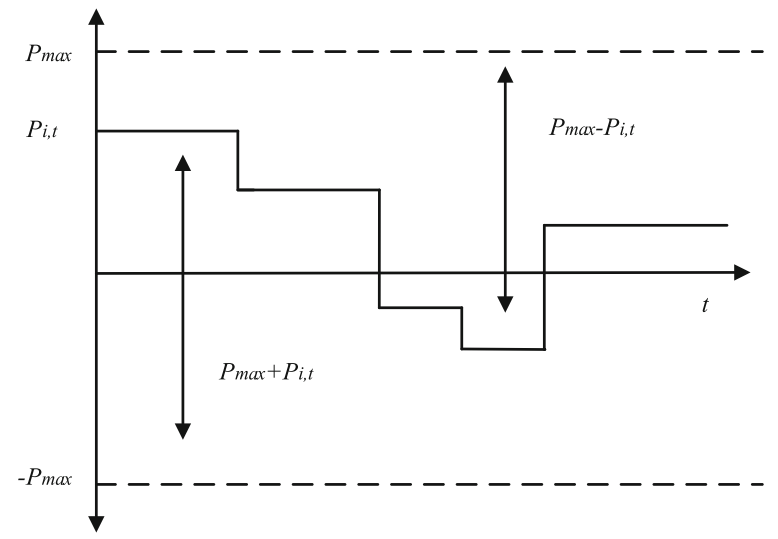

Fig. 6 Available capacity of an EV for regulation

present V2G power, as illustrated in Fig. 6. When the frequency deviation is negative, an EV will increase the discharging power or decrease the charging power to elevate system frequency. Therefore, the capacity for regulation up equals $P_{\max }$ plus $P_{i, t}$, where $P_{i, t}$ is the present power of the $i$ th EV at time $t$, as shown in Fig. 6. Similarly, the capacity for regulation down equals to $P_{\max }$ minus $P_{i}, t$. Thus, the capacity of an $\mathrm{EV}$ for regulation can be expressed as:

$$
\left\{\begin{array}{l}
\Delta P_{i, t}^{\mathrm{up}}=P_{\max }+P_{i, t} \\
\Delta P_{i, t}^{\text {down }}=P_{\max }-P_{i, t}
\end{array}\right.
$$

where $\Delta P_{i, t}^{\mathrm{up}}$ and $\Delta P_{i, t}^{\text {down }}$ are the capacities of the $i$ th EV for regulation up and regulation down at time $t$, respectively.

It was assumed that there is an EV aggregator with $n$ EV charging stations, each of which manages $m$ EVs. Therefore, in an EV charging station, the capacity for regulation can be calculated as:

$$
\left\{\begin{array}{l}
C_{j, t}^{\mathrm{up}}=\sum_{i=1}^{m} \Delta P_{i, t}^{\mathrm{up}} \\
C_{j, t}^{\text {down }}=\sum_{i=1}^{m} \Delta P_{i, t}^{\text {down }}
\end{array}\right.
$$

where $C_{j, t}^{\mathrm{up}}$ is the capacity for regulation up in an EV charging station at time $t$ and $C_{j, t}^{\text {down }}$ is the capacity for regulation down in an EV station at time $t$.

In the EV aggregator, the capacity for regulation can be calculated as:

$$
\left\{\begin{array}{l}
C_{t}^{\mathrm{up}}=\sum_{j=1}^{n} C_{j, t}^{\mathrm{up}} \\
C_{t}^{\text {down }}=\sum_{j=1}^{n} C_{j, t}^{\mathrm{down}}
\end{array}\right.
$$

where $C_{t}^{\text {up }}$ and $C_{t}^{\text {down }}$ are the capacities for regulation up and regulation down in the EV aggregator at time $t$, respectively.

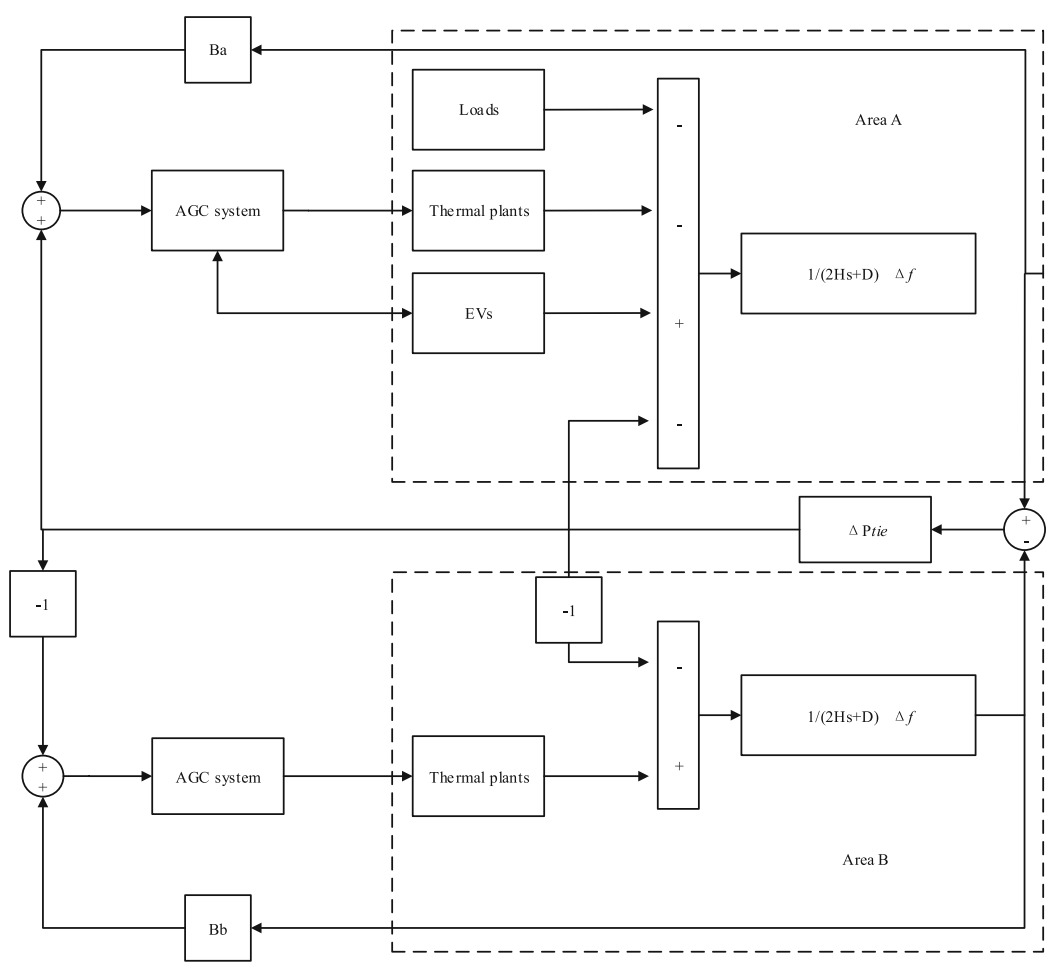

Fig. 7 A two-area interconnected power system 


\subsubsection{Hierarchical dispatch strategies}

As described in Section 2.1, hierarchical dispatch control includes the control center, EV aggregators, and EV charging stations. Respective dispatch strategies are proposed in this section for the three layers.

(1) Strategy in the control center

As shown in Figs. 4 and 5, high frequency signals of ACE or ARR can be dispatched to EVs. With high frequency signals, a control strategy for the control center shown in (7) is proposed to dispatch regulation tasks to an EV aggregator.

$$
P_{t}^{\mathrm{AG}}=\left\{\begin{array}{lc}
\max \left\{S_{t},-C_{t}^{\mathrm{up}}\right\} & S_{t}<0 \\
\min \left\{S_{t}, C_{t}^{\text {down }}\right\} & S_{t} \geq 0
\end{array}\right.
$$

In (7), $S_{t}$ is the high frequency signal of ACE or ARR at time $t$, and $P_{t}^{\mathrm{AG}}$ is the regulation task undertaking by an EV aggregator at time $t$.

(2) Strategy in the EV aggregator

Upon receiving the regulation task from the control center, the dispatch will be implemented from the EV aggregator to EV charging stations according to the following strategy:

$$
P_{j, t}^{\mathrm{chs}}= \begin{cases}P_{t}^{\mathrm{AG}} \times \frac{C_{j, t}^{\mathrm{up}}}{C_{t}^{\mathrm{up}}} & P_{t}^{\mathrm{AG}}<0 \\ P_{t}^{\mathrm{AG}} \times \frac{C_{j, t}^{\text {down }}}{C_{t}^{\text {down }}} & P_{t}^{\mathrm{AG}} \geq 0\end{cases}
$$

where $P_{j, t}^{\text {chs }}$ is the regulation task of the $j$ th EV charging station at time $t$.

\section{(3) Strategy in EV charging stations}

Table 1 Parameters of power systems

\begin{tabular}{lll}
\hline Parameters & Area-A & Area-B \\
\hline Maximum load capacity (MW) & 20,000 & 10,000 \\
Proportional and integral gains & 10.01 & 10.01 \\
Time constant for LFC (s) & 4 & 4 \\
Frequency bias factor (pu/Hz) & 0.15 & 0.075 \\
Inertia constant (pu.s) & 0.32 & 0.16 \\
Load damping coefficient (pu/Hz) & 0.04 & 0.02 \\
Synchronizing torque coefficient (Hz) & 0.04 & 0.04 \\
Dead band of primary frequency detection (s) & 0.033 & 0.033 \\
Time constant for frequency detection (s) & 0.1 & 0.1 \\
Communication delay (s) & 1 & 1 \\
Dead band of area control error (MW) & 10 & 10 \\
Ramp speed (MW/min) & 200 & 100 \\
\hline
\end{tabular}

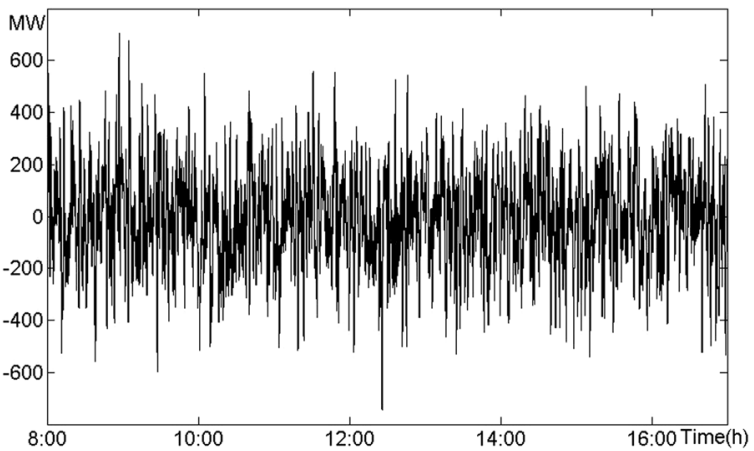

Fig. 8 Load fluctuation in a time series with $1 \mathrm{~s}$ intervals

For individual EVs in EV charging stations, the charging/discharging power is decided based on regulation dispatch and the present power. The regulation dispatch is distributed to individual EVs according to the following strategy:

$$
\Delta P_{i, t}^{\mathrm{EV}}= \begin{cases}P_{j, t}^{\mathrm{chs}} \times \frac{\Delta P_{i, t}^{\mathrm{up}}}{C_{j, t}^{\mathrm{up}}} & P_{j, t}^{\mathrm{chs}}<0 \\ P_{j, t}^{\mathrm{chs}} \times \frac{\Delta P_{i, t}^{\text {down }}}{C_{j, t}^{\text {down }}} & P_{j, t}^{\mathrm{chs}} \geq 0\end{cases}
$$

where $\Delta P_{i, t}^{\mathrm{EV}}$ is the power change for regulation of the $i$ th $\mathrm{EV}$ at time $t$.

Based on (9), the charging/discharging power of individual EVs can be expressed as:

$$
P_{i, t+1}^{\mathrm{EV}}=P_{i, t}^{\mathrm{EV}}+\Delta P_{i, t}^{\mathrm{EV}}
$$

where $P_{i, t+1}^{\mathrm{EV}}$ is the charging/discharging power of the $i$ th $\mathrm{EV}$ at time $t+1$.

Table 2 Simulation parameters of EVs

\begin{tabular}{ll}
\hline Parameters & \\
\hline EV Aggregator number & 1 \\
EV charging station number & 100 \\
EVs number in each charging station & 500 \\
Plug-in time (h) & Time $\sim$ N $(9,0.1)$ \\
Plug-out time (h) & Time N (16,0.1) \\
Initial SOC (pu) & SOC N $(0.4,0.05)$ \\
& SOC $\in[0.2,0.8]$ \\
Expected SOC (pu) & SOC N $(0.7,0.01)$ \\
& SOC $\in[0.4,0.9]$ \\
Battery capacity (kWh) & 32 \\
Maximum charging/discharging power (kW) & 7 \\
Maximum/minimum SOC (pu) & $0.9 / 0.2$ \\
\hline
\end{tabular}




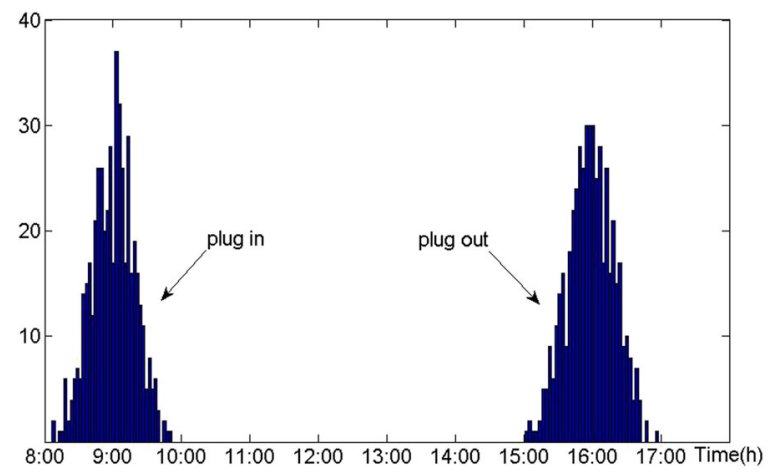

Fig. 9 Random integration and departure of EVs in a charging station

\section{The Simulation System}

\subsection{Interconnected power systems}

In order to verify the effectiveness of the proposed strategy, a two-area interconnected power system is used in Fig. 7 [12], and its detailed parameters are shown in Table 1. The load fluctuation follows a normal distribution with the mean of zero as illustrated in Fig. 8, and EVs are considered to integrate into the area-A.

\subsection{EV system module}

In this simulation, it considers an EV aggregator with 100 EV charging stations, each of which manages 500 EVs, as shown in Table 2. To describe the random behaviors of EVs integrated into the power grid, the normal distribution is used to simulate the plug-in time, the plug-out time, the initial SOC, and the expected SOC by the Latin Hypercube Sampling method.

An EV battery SOC level will be changed due to the charging/discharging in real time as:

$$
\mathrm{SOC}_{i, t}=\mathrm{SOC}_{i}^{\mathrm{in}}+\frac{\Delta E_{i}^{\mathrm{r}}}{E_{i}^{\mathrm{r}}}
$$

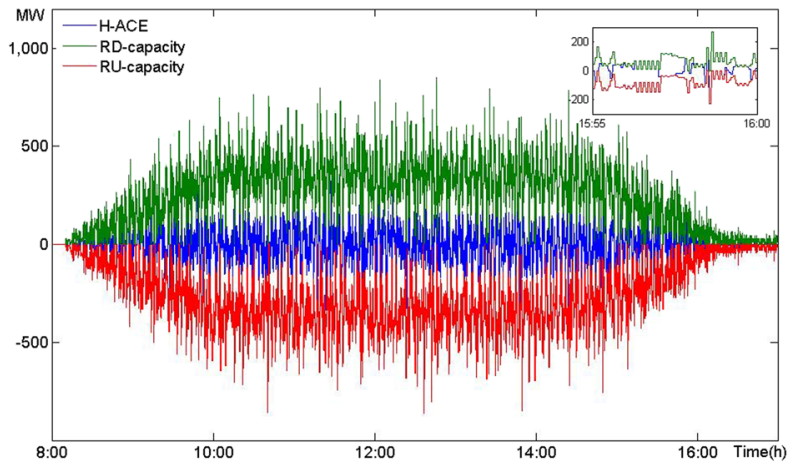

Fig. 10 The dispatch of H-ACE within the capacity of EVs for regulation

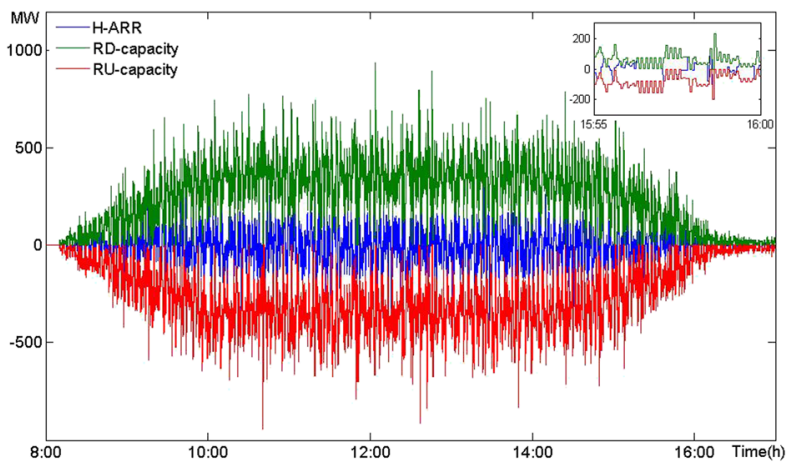

Fig. 11 The dispatch of H-ARR within the capacity of EVs for regulation

where $\Delta E_{i}^{\mathrm{r}}$ is the energy variation of the $i$ th EV battery and is given by:

$$
\Delta E_{i}^{\mathrm{r}}=\int_{0}^{t} P_{i, t}^{\mathrm{EV}} d t
$$

\section{Results and discussions}

\subsection{Simulation and discussion}

For simplicity, the regulation without EVs' participation is called as CS0. The proposed V2G strategies responding to high frequency components of $\mathrm{ACE}$ and ARR (H-ACE and H-ARR) are called as CS1 and CS2, respectively.

\subsubsection{Integration of EVs and dispatch}

While a normal distribution shown in Table 2 is considered for the plug-in and plug-out time of EVs, the randomness of the integration/departure of EVs into/ from the power grid is shown in Fig. 9. Because of this randomness, the regulation capacity of EVs, including regulation-up capacity (RU-capacity) and regulationdown capacity (RD-capacity), will increase from zero to the maximum at the plug-in time and decrease from the maximum to zero at the plug-out time, as shown in Figs. 9, 10 and 11.

Within EVs' regulation capacity, the regulation can be dispatched to EVs by either CS1 as in Fig. 10 or CS2 as in Fig. 11.

Table 3 The quality of grid frequency in area A

\begin{tabular}{llll}
\hline & CS0 & CS1 & CS2 \\
\hline $\operatorname{Max}(\mathrm{Hz})$ & 0.2132 & 0.1696 & 0.1654 \\
$\operatorname{Min}(\mathrm{Hz})$ & -0.2719 & -0.2379 & -0.2365 \\
RMS $(\mathrm{Hz})$ & 0.0575 & 0.0423 & 0.0405 \\
\hline
\end{tabular}


Table 4 The quality of ACE in area A

\begin{tabular}{llll}
\hline & CS0 & CS1 & CS2 \\
\hline Max (MW) & 846 & 687 & 693 \\
Min (MW) & -1131 & -971 & -966 \\
RMS (MW) & 232 & 172 & 163 \\
\hline
\end{tabular}

\subsubsection{Effect of EVs on grid frequency regulation}

While considering CSO, CS1, and CS2, the qualities of grid frequency and ACE are shown in Tables 3 and 4, respectively. As illustrated in Tables 3 and 4, compared with the case of $\mathrm{CSO}$, the frequency deviation and $\mathrm{ACE}$ can be suppressed more effectively by CS1 and CS2. This is because the high frequency components of ACE and ARR are dispatched to EVs, while EVs can perform better regulation of high frequency signals compared to traditional generation units.

\subsubsection{Power output reduction of generating units}

While considering EVs' participation in regulation, the high frequency components of ACE/ARR will be implemented by EVs, which results in reduction of regulation tasks undertaken by traditional generation units. Therefore, the power output of traditional generation units is reduced, as shown in Table 5.

In practice, EVs can undertake regulation of high frequency components of ACE/ARR due to the fast regulation and responding characteristics, while traditional generation units can improve their operation life by avoiding frequent regulation considering only the low frequency components of ACE/ARR.

\subsubsection{Influences of frequency regulation on EV battery}

In order to examine the influence of CS1 and CS2 on $\mathrm{EV}$ batteries, the index of power mileage $\mathrm{W}_{\mathrm{EV}}$ is defined as:

$$
\mathrm{W}_{\mathrm{EV}}=\sum_{i=1}^{N} \int_{0}^{t}\left|\Delta P_{i, t}\right| d t
$$

As shown in Fig. 12, using CS1 leads to higher power mileage than that of CS2, which means that CS1 exerts more influence on an EV battery than CS2 does. At the same time, Tables 3, 4 and 5 show that CS2 can achieve better regulation than CS1. Therefore, CS2 has more powerful potential in performing regulation than that of CS1.

Table 5 Power output reduction of generating unit

\begin{tabular}{llll}
\hline & CS0 & CS1 & CS2 \\
\hline Max (MW) & 405.84 & 400.81 & 400.91 \\
Min (MW) & -560.56 & -476.70 & -428.99 \\
RMS (MW) & 126.75 & 104.29 & 90.64 \\
\hline
\end{tabular}

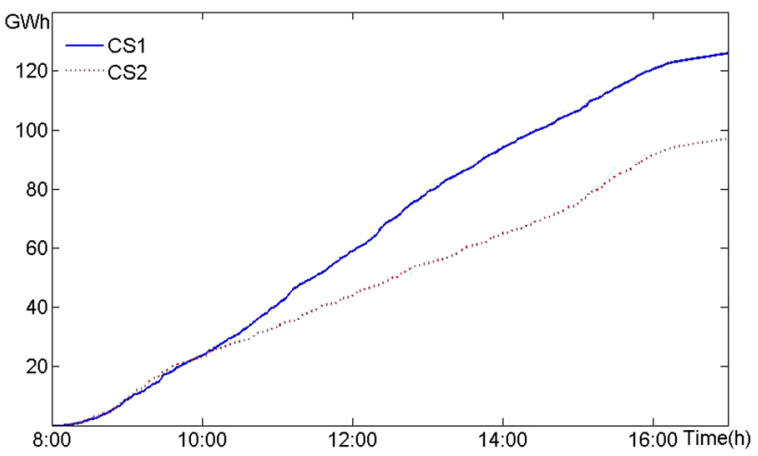

Fig. 12 The power mileages of an EV for CS1 and CS2

In order to satisfy EVs' charging demands, the charging of EVs are implemented with maximum charging power before departure. Since the duration for charging is budgeted at the plug-in time, charging demands can be achieved. For clarity, only one EV is considered for demonstration. For CS1 and CS2, the respective battery SOC levels of the EV are compared in Fig. 13. As seen, CS1 can maintain the battery SOC level at the plug-in time during regulation and achieve the expected battery SOC level after regulation. For CS2, although the battery SOC level deviates from the initial, the charging demand can be achieved before departure.

\section{Conclusion}

In this paper, the control strategies for EVs' participation in frequency regulation are investigated. While considering high frequency signals of ACE and ARR in the AGC systems, two different strategies are proposed to dispatch regulation tasks to EVs, where the EVs' regulation capacity is calculated based on the present power and the maximum power. In order to implement such a dispatch, a hierarchical control is implemented with an EV aggregator and EV charging stations. Finally, a twoarea interconnected power grid is modelled to examine the effectiveness of the proposed two strategies.

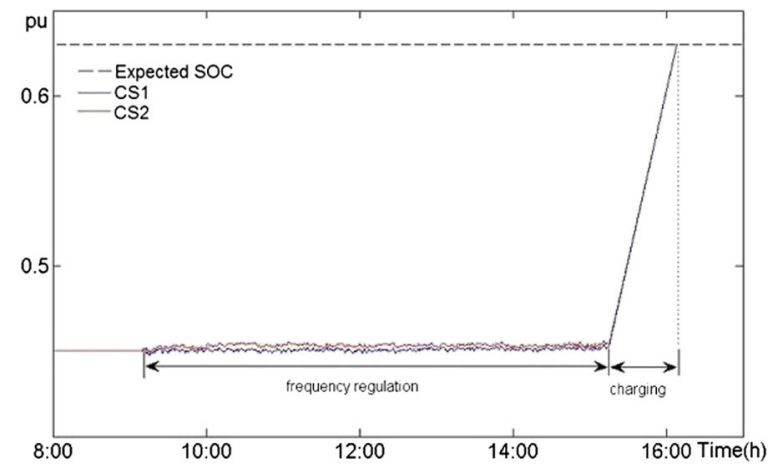

Fig. 13 Real-time battery SOC levels of an EV for CS1 and CS2 
Compared to the use of the high frequency signal of ACE, using the high frequency signal of ARR shows more advantages in performing regulation and reducing the power output of traditional generation units.

\section{Abbreviations}

ACE: Area control error; AGC: Automatic generation control; ARR: Area regulation requirement; EVs: Electric vehicles; H-ACE: The proposed V2G strategies responding to high frequency components of ACE; H-ARR: The proposed V2G strategies responding to high frequency components of ARR; RD-capacity: regulation-down capacity; RU-capacity: regulation-up capacity; SOC: State of charge; TBC: Tie-line frequency bias control; V2G: Vehicle to grid

\section{Funding}

This work was supported by Sino-US international Science and Technology Cooperation Project (Grant No. 2016YFE0105300).

\section{Availability of data and materials}

Please contact author for data requests.

\section{Authors' contributions}

$\mathrm{HL}$ conceived and designed the study. $\mathrm{KH}$ and $\mathrm{YY}$ performed the experiments and simulations. $\mathrm{KH}$ and $\mathrm{HL}$ wrote the paper. $\mathrm{HL}, \mathrm{KH}, \mathrm{YY}, \mathrm{HW}$ and SM reviewed and edited the manuscript. All authors read and approve the manuscript.

\section{Competing interests}

The authors declare that they have no competing interests.

\section{Author details}

'College of Electrical Engineering, Guangxi University, Nanning 530004,

China. ${ }^{2}$ China Electric Power Research Institute, Beijing 100192, China.

Received: 21 November 2017 Accepted: 23 April 2018

Published online: 21 May 2018

\section{References}

1. Li, C., Cao, Y., Zhang, M., Wang, J., Liu, J., Shi, H., et al. (2015). Hidden benefits of electric vehicles for addressing climate change[J]. Sci Rep, 5, 9213.

2. Green, R. C., Wang, L., \& Alam, M. (2011). The impact of plug-in hybrid electric vehicles on distribution networks: A review and outlook[J]. Renew Sustain Energy Rev, 15(1), 544-553.

3. Kempton, W., \& Letendre, S. E. (1997). Electric vehicles as a new power source for electric utilities[J]. Trans Res Part D Transp Environ, 2(3), 157-175.

4. Li, C., Cao, Y., Kuang, Y., \& Zhou, B. (2016). Influences of electric vehicles on power system and key Technologies of Vehicle-to-Grid[M]. Berlin Heidelberg: Springer.

5. Rogers, K. M., Klump, R., Khurana, H., Aquino-Lugo, A. A., \& Overbye, T. J. (2010). An authenticated control framework for distributed voltage support on the smart grid[J]. IEEE Trans Smart Grid, 1(1), 40-47.

6. Pavić, I., Capuder, T., \& Kuzle, I. (2015). Value of flexible electric vehicles in providing spinning reserve services[J]. Appl Energy, 157(November), 60-74.

7. Dallinger, D., Krampe, D., \& Wietschel, M. (2011). Vehicle-to-grid regulation reserves based on a dynamic simulation of mobility behavior[J]. IEEE Trans Smart Grid, 2(2), 302-313.

8. Luo, Z., Hu, Z., Song, Y., Xu, Z., \& Lu, H. (2013). Optimal coordination of plugin electric vehicles in power grids with cost-benefit analysis-Part i: Enabling techniques[J]. IEEE Trans Power Syst, 28(4), 3546-3555.

9. Fan, H., Jiang, L., Zhang, C. K., \& Mao, C. (2016). Frequency regulation of multi-area power systems with plug-in electric vehicles considering communication delays[J]. let Generation Transm Distrib, 10(14), 3481-3491.

10. Ma, T., \& Mohammed, O. (2013). Real-time plug-in electric vehicles charging control for $\mathrm{v} 2 \mathrm{~g}$ frequency regulation [C]. IECON 2013 -39th Annual Conference of the IEEE Industrial Electronics Society, Vienna, Austria, Nov. 2013.

11. Liu, H., Hu, Z., Song, Y., \& Lin, J. (2013). Decentralized vehicle-to-grid control for primary frequency regulation considering charging demands[J]. IEEE Trans Power Syst, 28(3), 3480-3489.

12. Liu, H., Hu, Z., Song, Y., Wang, J., \& Xie, X. (2015). Vehicle-to-grid control for supplementary frequency regulation considering charging demands[J]. IEEE Trans Power Syst, 30(6), 3110-3119.
13. Han, S., Han, S., \& Sezaki, K. (2010). Development of an optimal vehicle-to-grid aggregator for frequency regulation[J]. IEEE Trans Smart Grid, 1(1), 65-72.

14. Kempton, W., \& Tomić, J. (2005). Vehicle-to-grid power fundamentals: Calculating capacity and net revenue[J]. J Power Sources, 144(1), 268-279.

15. Masuta, T., \& Yokoyama, A. (2012). Supplementary load frequency control by use of a number of both electric vehicles and heat pump water heaters [J]. IEEE Trans Smart Grid, 3(3), 1253-1262.

16. Han, S., Han, S., \& Sezaki, K. (2011). Estimation of achievable power capacity from plug-in electric vehicles for $v 2 \mathrm{~g}$ frequency regulation: Case studies for market participation[J]. IEEE Trans Smart Grid, 2(4), 632-641.

17. Lam, A. Y. S., Leung, K. C., \& Li, V. O. K. (2017). Capacity estimation for vehicle-to-grid frequency regulation services with smart charging mechanism[J]. IEEE Trans Smart Grid, 7(1), 156-166.

18. Zhang, H., Hu, Z., Xu, Z., \& Song, Y. (2017). Evaluation of achievable vehicle-to-grid capacity using aggregate PHEV model[J]. IEEE Trans Power Syst, 32(1), 784-794.

19. Hu, Z., Xie, X., Fang, F., Zhang, J., \& Song, Y. (2014). Research on automatic generation control strategy incorporating energy storage resources[J]. Proc CSEE, 34(29), 5080-5087.

\section{Submit your manuscript to a SpringerOpen ${ }^{\circ}$ journal and benefit from:}

- Convenient online submission

- Rigorous peer review

- Open access: articles freely available online

- High visibility within the field

- Retaining the copyright to your article

Submit your next manuscript at $>$ springeropen.com 